\section{Subarachnoid haemorrhage? Think again}

\section{Cjien-Ting Liu, ${ }^{1}$ Chien-Chin Hsu, ${ }^{1,2}$ Kuo-Tai Chen ${ }^{1,3}$}

\section{'Emergency Department, Chi-Mei Medical Center, Tainan; ${ }^{2}$ Department of Biotechnology, Southern Tainan University of Technology; ${ }^{3}$ Department of Emergency Medicine, Taipei Medical University, Taipei}

A 69-year-old male patient was admitted to a hospital for acute cerebral infarction. During hospitalisation, he developed pneumonia, septic shock, bowel ischemia, and acute renal failure, and was transferred to our hospital for further treatment. On arrival, he underwent another non-contrast enhanced cranial computed tomography (CT), which suspected the presence of subarachnoid haemorrhage (SAH) (Figure 1). We then consulted a neurosurgeon urgently but additional image studies were avoided to prevent contrast agent-related nephrotoxicity. Subsequent radiology confirmed that the cranial CT displayed pseudo-SAH, which may have resulted from hypoxic encephalopathy-induced severe cerebral oedema.

The patient was administered broadspectrum antibiotics, aggressive haemodynamic support for septic shock, and mannitol infusion for the cerebral oedema. However, the sepsis progressed and he died 6 days after admission from multiple organ failure.

Marked cerebral oedema may develop in patients with hypoxic encephalopathy, severe head trauma, meningitis, postradiation therapy, and hypervolemia. ${ }^{1,2}$ Several studies have reported that cranial CT of such patients sometimes reveals increased attenuation along the basal cisterna or cortical sulci, which mimics SAH. ${ }^{1-3}$

The key to differentiating true and pseudo-SAH is identifying the presence of severe cerebral oedema in the absence of a large amount of subarachnoid blood or a substantial parenchymal haematoma on a
Correspondence: Kuo-Tai Chen, Emergency Department, Chi-Mei Medical Center

901 Chung-Hwa Road, Yung Kang, Tainan 710, Taiwan

E-mail: 890502@mail.chimei.org.tw

Key words: subarachnoid haemorrhage; pseudosubarachnoid haemorrhage; cerebral oedema; computed tomography; misdiagnosis.

Contributions: CTL: manuscript drafting $\mathrm{CCH}$ : art work of the figure. KTC: manuscript revision.

Conflict of interest: the authors declare no potential conflict of interest.

Received for publication: 3 March 2017

Revision received: 20 September 2017.

Accepted for publication: 22 September 2017

This work is licensed under a Creative Commons Attribution 4.0 License (by-nc 4.0).

(C) Copyright C-T. Liu et al., 2017

Licensee PAGEPress, Italy

Emergency Care Journal 2017; 13:6681

doi:10.4081/ecj.2017.6681
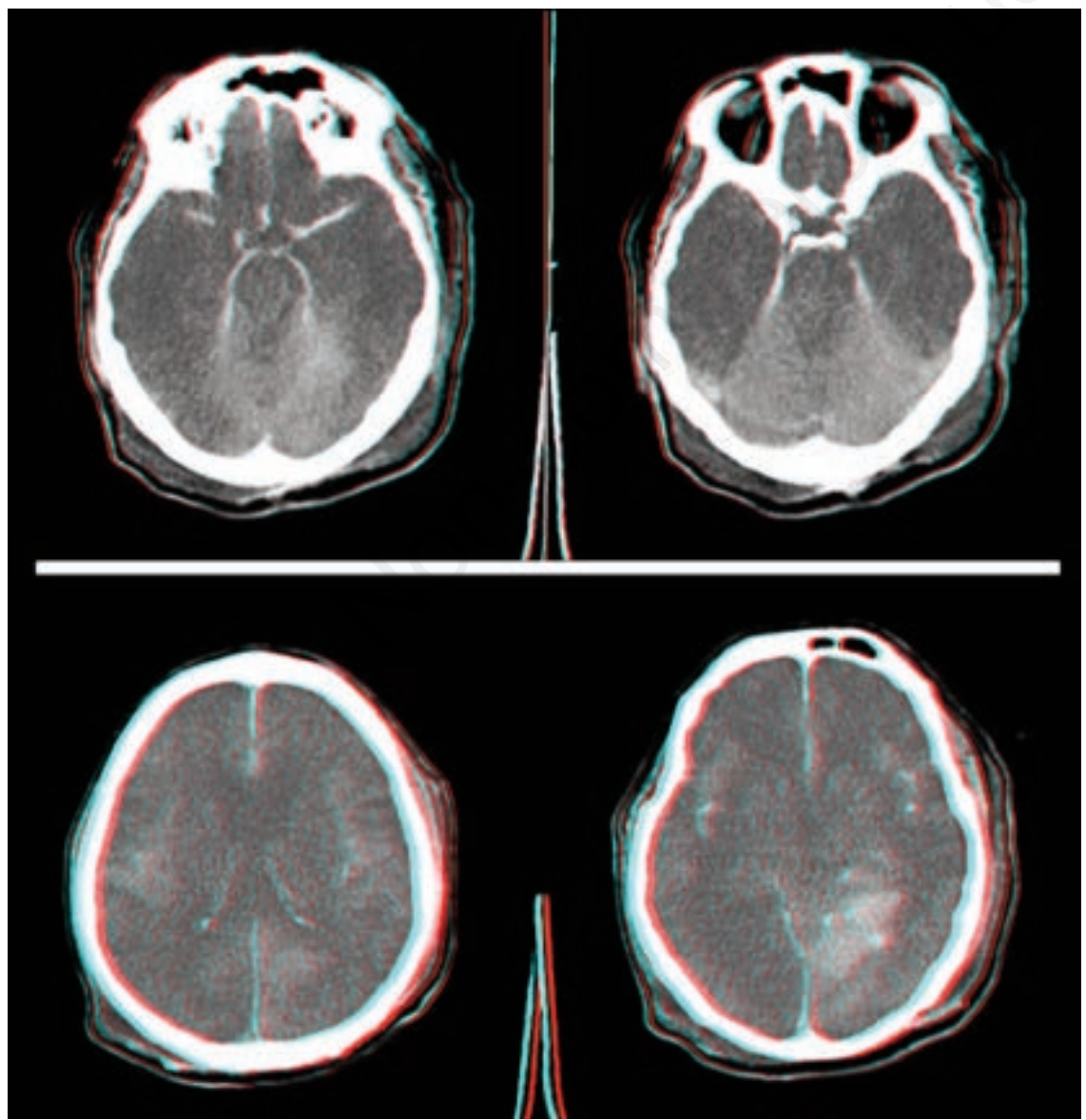

Figure 1. Cranial computed tomography scan revealing increased attenuation along the basal cisterna or cortical sulci, which mimics SAH. In the absence of a large amount of subarachnoid blood or a parenchymal haematoma, images of pseudo-SAH show the marked effacement of sulci and basal cisterns, invisible ventricles, and poor grey-white matter differentiation. cranial CT scan. Cranial CT of pseudo-SAH will show the marked effacement of sulci and basal cisterns, slit-like ventricles, and poor grey-white matter differentiation. ${ }^{1,2}$ Furthermore, the distribution of blood along the fissure and sulci exhibits irregular hyperdensity in true SAH, whereas it displays a smoother line in pseudo-SAH.

\section{References}

1. Avrahami E, Katz R, Rabin A, Friedman V. CT diagnosis of non-traumatic haemorrhage in patients with brain edema. Eur J Radiol 1998;28:222-5.

2. Rabinstein AA, Pittock SJ, Miller GM, et al. Pseudosubarachnoid haemorrhage in subdural haematoma. J Neurol Neurosurg Psychiatry 2003;74:1131-2.

3. Yuzawa H, Higano S, Mugikura S, et al. Pseudo-subarachnoid haemorrhage found in patients with postresuscitation encephalopathy: characteristics of CT findings and clinical importance. Am J Neuroradiol 2008;29:1544-9. 